\title{
Autonomic nervous system dysfunction and fatigue in multiple sclerosis: common pathophysiology or spurious association?
}

\author{
Juan Manuel Racosta ${ }^{1,2}$ \\ Received: 7 November 2018 / Accepted: 9 November 2018 / Published online: 17 November 2018 \\ c) Springer-Verlag GmbH Germany, part of Springer Nature 2018
}

Keywords Multiple sclerosis $\cdot$ Demyelinating disorders $\cdot$ Autoimmune disorders $\cdot$ Fatigue $\cdot$ Dysautonomia

An emerging body of literature suggests that many of the disabling symptoms experienced by patients with multiple sclerosis, including fatigue, are the result of cardiovascular autonomic dysregulation. Clinicians treating patients with multiple sclerosis intuitively embrace this literature and many of the potential mechanisms suggested, given the overwhelming similarity between symptoms of cardiovascular autonomic dysfunction and those reported by these patients. Within this realm of possible associations, discerning between common pathophysiology and spurious association becomes the foremost and most challenging goal.

That multiple sclerosis and related disorders can cause severe urinary autonomic dysfunction is well documented in the literature [6]. However, the design of clinical research to define whether, in multiple sclerosis, cardiovascular autonomic dysfunction is contributing to fatigue is plagued by several difficulties. First, fatigue is an "umbrella term" which, despite countless efforts, is still often confounded with other common symptoms such as sleepiness, psychomotor slowing, or lightheadedness. Second, the wide variety of putative mechanisms that have been proposed are, for the most part, plausible and likely coexist. These mechanisms are related to either increased energetic demands or decreased metabolic supply. This overlap often makes statistical analysis complex and misleading. Third, and most importantly, standard autonomic function testing, based on peripheral autonomic measurements only, might be inaccurate indicators of central autonomic changes where independent mechanisms, such as cerebral autoregulation, take

Juan Manuel Racosta

juanracosta@hotmail.com

1 Division of Neurology, Health Sciences Centre, University of Manitoba, Winnipeg, MB, Canada

2 Multiple Sclerosis Clinic, Health Sciences Centre, GF543-820 Sherbrook St., Winnipeg, MB R3A 1R9, Canada place. In other words, standard autonomic tests alone have low internal validity for symptoms associated with central hypoperfusion in the absence of obvious blood pressure abnormalities. This last constraint is exemplified by the frequent presence of orthostatic intolerance in patients with normal autonomic reflexes.

In this issue of Clinical Autonomic Research, Krbot Skorić and colleagues studied 70 patients with multiple sclerosis and found a significant association between fatigue (as defined by the Modified Fatigue Impact Scale) and autonomic symptoms defined by a validated questionnaire (COMPASS-31) [4]. Interestingly, higher levels of fatigue were associated with older age, smaller bradycardic response in phase IV of the Valsalva maneuver (Valsalva ratio), and smaller sweating volumes. In turn, when analyzing autonomic symptoms, the more "peripheral" domains of COMPASS-31, namely vasomotor, secretomotor, gastrointestinal, and pupillomotor symptoms, were correlated with cardiovascular autonomic reflexes and sudomotor dysfunction, but the "central" symptom domain (i.e., orthostatic intolerance) was not. These associations led the authors to conclude that peripheral autonomic nervous system dysregulation has a role in the pathophysiology of fatigue in multiple sclerosis.

Although the authors propose that cardiovascular autonomic dysregulation causes fatigue, their research eloquently highlights the limitations in the field. The conclusions of this appropriately designed, sufficiently powered, and judiciously analyzed study appear to be confounded by the effect of aging. Older age is associated with fatigue and with less reactive vagal reflexes. Thus, the suggestion that peripheral impairment significantly explains fatigue in a disease resulting from evident central nervous system dysfunction is seemingly misleading. In other words, this problem boils down to the investigation of poorly defined variables using methods that are not valid for the full assessment of central autonomic physiology. 
A feasible solution to these methodological difficulties is the inspection of autonomic regulation of cerebral hemodynamics, which gained some prominence in recent literature. By means of simultaneous recording of blood pressure, cerebral blood flow velocity, and cerebral oxy- and deoxyhemoglobin concentration, the capacity of cerebral vasculature to regulate blood flow can be estimated [3]. Autonomic receptors, both sympathetic and parasympathetic, have been shown to have a salient role in dynamic cerebral autoregulation, especially in circumstances of normal blood pressure (active range of dynamic autoregulation) [2]. These methods represent a logical direction in the assessment of the unequivocal effects of autonomic dysregulation on fatigue and other central symptoms. Patients with multiple sclerosis represent an optimal population to apply these methods, since the majority of them have no abnormalities in blood pressure control. They often present with symptoms suggestive of autonomic dysregulation, and do not pose the technical difficulties with the use of transcranial Doppler that are seen in older individuals [5]. In addition, robust evidence of cerebral hypoperfusion in patients with multiple sclerosis has emerged with the extensive use of newer MRI techniques, reinforcing the hypothesis of central vascular impairment [1]. Surprisingly, despite the existence of laboratories with advanced autonomic testing capabilities, scarce research using these methods has been carried out to date. Therefore, in the advent of the adolescent era of central autonomic nervous system assessment, studies such as that of Krbot Skorić and colleagues should be reformulated to include the evaluation of cerebral hemodynamics. By doing so, we might be able to answer whether, in patients with multiple sclerosis, autonomic symptoms and fatigue share a common pathophysiology, or if their relationship is just a spurious finding.
Funding None.

\section{Compliance with ethical standards}

Conflict of interest The author declares that they have no conflict of interest.

\section{References}

1. D'Haeseleer M, Hostenbach S, Peeters I, Sankari SE, Nagels G, De Keyser J, D'Hooghe MB (2015) Cerebral hypoperfusion: a new pathophysiologic concept in multiple sclerosis? J Cereb Blood Flow Metab 35:1406-1410

2. Hamner JW, Tan CO (2014) Relative contributions of sympathetic, cholinergic, and myogenic mechanisms to cerebral autoregulation. Stroke 45:1771-1777

3. Jakimovski D, Topolski M, Kimura K, Marr K, Gandhi S, Ramasamy DP, Bergsland N, Hagemeier J, Weinstock-Guttman B, Zivadinov R (2018) Abnormal venous postural control: multiple sclerosis-specific change related to gray matter pathology or age-related neurodegenerative phenomena? Clin Auton Res. https ://doi.org/10.1007/s10286-018-0555-6

4. Krbot Skoric M, Crnosija L, Adamec I, Barun B, Gabelic T, Smoljo T, Stanic I, Pavicic T, Pavlovic I, Drulovic J, Pekmezovic T, Habek M (2018) Autonomic symptom burden is an independent contributor to multiple sclerosis related fatigue. Clin Auton Res. https://doi.org/10.1007/s10286-018-0563-6

5. Norcliffe-Kaufmann L, Galindo-Mendez B, Garcia-Guarniz AL, Villarreal-Vitorica E, Novak V (2018) Transcranial Doppler in autonomic testing: standards and clinical applications. Clin Auton Res 28:187-202

6. Sakakibara R (2018) Neurogenic lower urinary tract dysfunction in multiple sclerosis, neuromyelitis optica, and related disorders. Clin Auton Res. https://doi.org/10.1007/s10286-018-0551-x 